\title{
Pure air-plasma bullets propagating inside microcapillaries and in ambient air
}

\author{
Deanna A Lacoste ${ }^{1}$, Anne Bourdon ${ }^{2}$, Koichi Kuribara ${ }^{3}$, \\ Keiichiro Urabe ${ }^{3}$, Sven Stauss ${ }^{3}$, and Kazuo Terashima ${ }^{3}$ \\ ${ }^{1}$ Clean Combustion Research Center, King Abdullah University of Science and \\ Technology, Thuwal 23955-6900, Saudi Arabia \\ ${ }^{2}$ Laboratory of Plasma Physics (LPP), UMR7648, Ecole Polytechnique, UPMC, \\ Université Paris Sud 11, CNRS, Palaiseau, France \\ ${ }^{3}$ Department of Advanced Materials Science, Graduate School of Frontier Sciences, \\ The University of Tokyo, 5-1-5 Kashiwanoha, Kashiwa-shi, Chiba 277-8562, Japan \\ E-mail: deanna.lacoste@kaust.edu.sa
}

\begin{abstract}
.
This paper reports on the characterization of air-plasma bullets in microcapillary tubes and in ambient air, obtained without the use of inert or noble gases. The bullets were produced by nanosecond repetitively pulsed (NRP) discharges, applied in a dielectric barrier discharge (DBD) configuration. The anode was a tungsten wire with a diameter of $50 \mu \mathrm{m}$, centered in the microcapillary, while the cathode was a silver ring, fixed on the outer surface of the fused silica tube. The effect of applied voltage and inner diameter of the microcapillary tube on the plasma behavior were investigated. Inside the tubes, while the topology of the bullets seems to be strongly dependent on the diameter, their velocity is only function of the applied-voltage amplitude. In ambient air, the propagation of air-bullets with a velocity of about $1.25 \times 10^{5} \mathrm{~ms}^{-1}$ is observed.
\end{abstract}

\section{Introduction}

Guided streamers, so-called plasma bullets, in ambient air, have been studied extensively in the last decade [1-5]. The main application fields of these non-equilibrium plasmas propagating out of the interelectrode region are bio-medicine [6-8] and surface treatment [9-11]. These applications are temperature sensitive, therefore, the control of gas heating by the plasma generation is a key point. A convenient way to create a non-thermal plasma at atmospheric pressure is the use of noble or inert gases, in order to reduce the breakdown voltage, in a dielectric barrier discharge (DBD) configuration, which restricts the conductive current. Thus, in the large majority of previous studies, the discharges were generated in pure or mixture-containing helium [1-5], argon [12,13], neon [14,15], or krypton [16]. However, the high cost of these gases could be an issue for some applications. 
On the other hand, in air or in air-containing mixtures, non-thermal plasma discharges at atmospheric pressure have also been extensively studied for numerous applications such as flue gas treatment [17], plasma-assisted combustion [18] or biomedical applications [19]. However, to our best knowledge, the generation of pure air-plasma bullets by non-thermal plasma discharges at atmospheric pressure has never been reported. This is the purpose of the present study.

\section{Experimental setup}

The air-plasma bullets were produced by nanosecond repetitively pulsed (NRP) discharges in a DBD configuration. Figure 1 presents the two experimental configurations used in this study. The anode is a tungsten wire of $50-\mu \mathrm{m}$ diameter, centered in the microcapillary, while the cathode is a silver ring, fixed on the outer surface of the tube. In order to avoid parasitic discharges outside the microcapillary, a 1-mm thick insulation layer covers the silver ring. The tubes used are out of fused silica (IDEX Corporation). They have inner diameters (IDs) of 100, 150, 300 and $500 \mu \mathrm{m}$, i.e. in the same range as the typical diameter of discharges in air at atmospheric pressure $[20,21]$. The corresponding thickness of the tubes are comprised between 115 and $200 \mu \mathrm{m}$. The microcapillary is fed with a 4 -sccm flow of air at atmospheric pressure, inducing a flow velocity always slower than $10 \mathrm{~ms}^{-1}$. The injected gas is dried air, obtained by compression and refrigeration process (down to $233 \mathrm{~K}$ ). The gap distance is kept constant at $1.35 \pm 0.15 \mathrm{~mm}$. For studying the bullets propagation inside the tube, the length of the microcapillary after the cathode is fixed at $5 \mathrm{~cm}$ [see figure 1(a)]. For the generation of the plasma bullets in ambient air [see figure 1(b)], the length of the tube after the silver ring is about $1 \mathrm{~mm}$. In order to avoid propagation of streamers on the outer surface of the microcapillary, a square plate of teflon $\left(2 \times 2 \mathrm{~cm}^{2}\right.$, thickness $0.8 \mathrm{~mm}$ ) is fixed perpendicularly to the tube exit.

The high-voltage pulses of $10-n s$ duration and $5.2-8.2 \mathrm{kV}$ amplitude, at a repetition frequency of $1 \mathrm{kHz}$, were generated by a solid-state pulse generator (FPG 10-30NM10, FID GmbH). Voltage and current across the electrodes were measured with appropriate probes (a Tektronix $75 \mathrm{MHz}$ high-voltage probe, model P6015A, and a Pearson coil, model 2877). Both signals were recorded simultaneously with a $500 \mathrm{MHz}$ bandwidth oscilloscope (Agilent Technology DSOX3054A). The energy deposition per pulse was determined from these electrical measurements, following the method detailed by Pai [22] and summarized elsewhere [23]. First, a synchronization between the curent and voltage signals was performed. Indeed, any cable or probe affects differently the lag time between the discharge events and measurements. For nanosecond duration pulses, this lag time is significant and a synchronization is necessary. For this purpose, in this study, the current waveforms were shifted by $6.5 \mathrm{~ns}$. Then, the current and voltage signals were multiplied and integrated to obtain the energy deposition per pulse. An example of synchronized voltage and current waveforms, and the corresponding energy per pulse, is presented in figure 2. The beginning of the high-voltage pulse defines the 
reference time, $t_{0}$. The ringing in both the voltage and current signals is due to parasitic impedance and capacitance in the electric circuit. The subsequent resulting error on the energy determination is estimated to be of about $20 \%$. Therefore, the energy per pulse should be taken only as an indicator, not allowing further analysis.

The propagation velocity of the bullets was determined with the aid of an intensified charge coupled device (ICCD) camera (Princeton Instruments PIMax, $1024 \times 1024$ pixels $^{2}$ ), equipped with a zoom lens (Thorlabs, Navitar 1-50486). Each image was obtained by accumulating over 20,000 events. The exposure time was $2 \mathrm{~ns}$. The images were synchronized with the discharges using a function generator (Tektronix AFG 3021). The jitter between two pulses was very low, less than $0.5 \mathrm{~ns}$, and the synchronisation accuracy between the camera and the plasma generator was about $0.2 \mathrm{~ns}$. Taking into account the propagation speed of the observed phenomenon, the scattering effect due to accumulation and synchronization can be neglected. For a given image, the position of the discharge front was defined as the right part of the bullet where the pixel intensities were about $20 \%$ of the maximum intensity of this particular image. In order to facilitate visualization of the plasma, the greyscale images have been mapped to a color lookup table.

The gas temperature of the plasma discharge has been investigated by optical emission spectroscopy (OES) analysis. The optical setup comprised an Acton SpectrPro320i spectrometer (focal length $320 \mathrm{~mm}$, grating of $1200 \mathrm{gr} / \mathrm{mm}$, blazed at $300 \mathrm{~nm}$ ) fitted with a $1024 \times 1024$ pixels $^{2}$ ICCD camera (Princeton Instrument PIMax) and an imaging fiber adapter (Acton FC-446-010) combined with an optical fiber (Acton LG-4550-020). The full width at half maximum (FWHM) of the slit function was $0.83 \mathrm{~nm}$. All the light from the plasma zone, including the interelectrode area, was collected. Each spectrum was obtained by accumulating over 100,000 events. The exposure time was $2 \mathrm{~ns}$, synchronized with the plasma discharges, by using a function generator (Tektronix AFG 3021).

\section{Results and discussion}

Figures 3 (a)-(f) present the discharge dynamics out of the inter electrode region for a $100-\mu \mathrm{m}$ ID microcapillary. The applied voltage was $7.2 \mathrm{kV}$ and the energy per pulse $85 \mu \mathrm{J}$. The fast-moving discharges obtained have a luminous front filling the whole section of the microcapillary. They propagate from the cathode area to the outlet of the tube, in the direction of the air flow. This behavior, similar to plasma bullets in helium, allows us to call these discharge fronts, air-plasma bullets. Figure 3 (g) shows air-plasma bullets obtained with a 500- $\mu \mathrm{m}$ ID microcapillary. In this case, the discharges propagate at the vicinity of the surface. This effect of the microcapillary diameter on the topology has already been observed by Jansky et al. [24], for similar discharges propagating between electrodes. They explained that, in air, due to the relatively high permittivity of the capillary material, the electric field increases close to the surfaces. Then, the discharges naturally propagate close to the surfaces. However, when the 
diameter of the microcapillary is of the order of the typical size of the discharge, it is constricted by the surfaces and appears homogeneous. These effects could also explain our present results.

The duration of light emission from the plasma region is about $25 \mathrm{~ns}$. Knowing the delay between two images, the bullets velocity can be determined. Figure 4 presents the influence of the microcapillary ID on the temporal evolution of the bullet velocity. The reproducibility of the experiments has been verified by repeating three times each measurement. The error bars represent the uncertainty in estimating the front of the discharges from the ICCD images. These results have been obtained for an applied voltage of $8.2 \mathrm{kV}$ and an energy per pulse of $115 \mu \mathrm{J}$. At $1 \mathrm{kHz}$, the corresponding average power is then $115 \mathrm{~mW}$. First, even if the shape of the bullets depends on the microcapillary ID, this parameter does not seem to affect their velocity. Indeed, regarding the measurement uncertainty, for IDs from 100 to $500 \mu \mathrm{m}$, between 8 and $14 \mathrm{~ns}$ after $\mathrm{t}_{0}$, the velocity of the bullets is about $4 \times 10^{5} \mathrm{~ms}^{-1}$. Then, it decreases rapidly to less than $1 \times 10^{5} \mathrm{~ms}^{-1}$, and finally the bullets vanish. This range of velocities corresponds to the one obtained by Jansky et al. [24] for similar conditions, between electrodes.

The effect of the applied voltage has been investigated for a $100-\mu \mathrm{m}$ ID microcapillary. Figure 5 shows that the maximum velocity of the bullets strongly depends on the applied-voltage amplitude. For a delay of $10 \mathrm{~ns}$ after the beginning of the pulses, the velocity of the air-plasma bullets is four times higher at $8.2 \mathrm{kV}$ than at $5.2 \mathrm{kV}\left(5.2 \times 10^{5}\right.$ and $1.2 \times 10^{5} \mathrm{~ms}^{-1}$, respectively). This behavior is in agreement with previous results obtained in helium [25]. A 5-mm propagation length of the air-plasma bullets inside the microcapillary has been obtained for an applied voltage of $8.2 \mathrm{kV}$. This length could probably be increased by applying longer high-voltage pulses (typically a few hundreds of nanoseconds, as in references $[25,26]$ ). Finally, the gas temperature has been determined from spectroscopic analysis of the $\mathrm{N}_{2}(\mathrm{C}-\mathrm{B})$ transition (obtained with a methodology detailed elsewhere [23]). Figure 6 presents an example of experimental spectrum, obtained for a 100- $\mu \mathrm{m}$ ID microcapillary and an applied voltage of $8.2 \mathrm{kV}$, at the plasma-emission peak, and associated simulations performed with the SPECAIR software $[27,28]$ for a vibrational temperature of $3500 \mathrm{~K}$, and rotational temperatures, $\mathrm{T}_{\text {rot }}$, of 300, 400 and $500 \mathrm{~K}$. Thus, for this experimental condition, the heating of the gas by the discharges has been found to be about $100 \mathrm{~K}$. During the $25 \mathrm{~ns}$ of the $\mathrm{N}_{2}(\mathrm{C}$ B) emission, all the recorded spectra were similar, with no significant temperature evolution. Thus, the thermal impact of the discharges seems to be reasonable. However, further investigation would be necessary to conclude about the temperature of these airplasma bullets.

Hofmann et al. [29] have shown the propagation of pure argon-plasma bullets in an argon atmosphere, at atmospheric pressure. They explained this result by a memory effect due to previous pulses. Indeed, by adjusting the frequency of the pulses and the flow velocity, guiding of subsequent streamers is possible, leading to the propagation of plasma bullets. In pure helium, due to a combined low electronic density in the head of 
the streamer and slow propagation of the ionization front [30], they did not observe the propagation of bullets, but a diffuse and homogeneous plasma.

In order to determine the plasma behavior in pure air, a similar setup [see figure $1(\mathrm{~b})$ ], with a $150-\mu \mathrm{m}$ ID microcapillary opening on a dielectric plane, was used. Figure 7 presents images of the observed discharge behavior. Air-plasma bullets clearly propagate in ambient air, from the outlet of the capillary, in the direction of the main flow. Considering the low speed of this flow (less than $10 \mathrm{~ms}^{-1}$ ), this behavior cannot be explained as in the work of Xian et al. [31] by a fast convection of the plasma generated between the electrodes. In air, the memory effect could also be responsible for the guidance of the streamers. Indeed, the pre-ionization level of the flow (mainly $\mathrm{O}_{2}^{-}$and $\mathrm{O}_{4}^{+}$at room temperature $[32,33]$ ), induced by the previous pulses, could be high enough to generate a confined plasma propagating in the air-jet. Tholin et al. [34] have shown that for similar NRP discharges in ambient air, taking into account a detailed chemistry of charged species during the inter-pulse time, the ion density, $1 \mathrm{~ms}$ after a pulse, remains high, about $10^{10} \mathrm{~cm}^{-3}$.

In parallel, a surface discharge develops on the dielectric plate, in a quasi-perfect circular shape. For $7.2-\mathrm{kV}$ applied voltage, a circle of about 6 - $\mathrm{mm}$ diameter is formed in $10 \mathrm{~ns}$, which correspond to an average propagation speed of $1.4 \times 10^{6} \mathrm{~ms}^{-1}$. One reason for the development of this surface discharge could be a local increase in the electric field close to the teflon surface, but to explain this effect, further investigations are required.

Figure 8 presents the influence of the applied voltage on the air-plasma bullets velocity in ambient air. Unlike the plasma confined in microcapillaries, in the range of 6.2 to $8.2 \mathrm{kV}$, the bullets velocity is not significantly affected by the applied voltage, with an average propagation velocity of $1.25 \times 10^{5} \mathrm{~ms}^{-1}$. Typically, a $4-\mathrm{mm}$ propagation length of the air-plasma bullets in ambient air has been obtained for $7.2-\mathrm{kV}$ applied voltage.

\section{Conclusion}

This study puts forward the generation of air-plasma bullets at atmospheric pressure inside dielectric microapillaries and in ambient air, without the use of inert or noble gases. For inner diameters of the dielectric tubes in the range of the typical diameter of the discharges, actually between 100 and $500 \mu \mathrm{m}$, electrically-driven streamer propagation outside the electrode gap has been observed. It has also been shown that, even if the shape of the bullets depends on the microcapillary inner diameter, this parameter does not significantly affect their velocity. Conversely, the plasma bullets velocity depends on the applied voltage inside the tubes but not much in ambient air.

\section{Acknowledgments}

This research was supported by a Grant-in-Aid for Scientific Research on Innovative Areas, Grant No 21110002, from the Ministry of Education, Culture, Sports, Science 
and Technology of Japan, and by a Grant-in-Aid for Scientific Research (A), Grant No 24246120, from the Japan Society for the Promotion of Science.

\section{References}

[1] Teschke M, Kedzierski J, Finantu-Dinu E, Korzec D and Engemann J 2005 IEEE Trans. Plasma Sci. 33 310-311

[2] Lu X and Laroussi M 2006 J. Appl. Phys. 100063302

[3] Urabe K, Morita T, Tachibana K and Ganguly B 2010 J. Phys. D: Appl. Phys. 43095201

[4] Jansky J, Algwari Q, O'Connell D and Bourdon A 2012 IEEE Trans. Plasma Sci. 40 2912-2919

[5] Lu X, Laroussi M and Puech V 2012 Plasma Sources Sci. Technol. 21034005

[6] O'Connell D, Cox L, Hyland W, McMahon S, Reuter S, Graham W, Gans T and Currell F 2011 Appl. Phys. Lett. 98043701

[7] Hoentsch M, von Woedtke T, KD W and Nebe J 2012 J. Phys. D: Appl. Phys. 45025206

[8] Park G, Park S, Choi M, Koo I, Byun J, Hong J, Sim J, Collins G and Lee J 2012 Plasma Sources Sci. Technol. 21043001

[9] Babayan S, Jeong J, Park J, Selwyn G and Hicks R 1998 Plasma Sources Sci. Technol. 7286

[10] Xu L, Liu P, Zhan R, Wen X, Ding L and Nagatsu M 2006 Thin Solid Films 506-507 400-403

[11] Ito Y, Urabe K, Takano N and Tachibana K 2008 Appl. Phys. Express 1067009

[12] Kim H, Brockhaus A and Engemann J 2009 Appl. Phys. Lett. 95211501

[13] Bussiahn R andKindel E, Lange H and Weltmann K 2010 J. Phys. D: Appl. Phys. 43165201

[14] Robert E, Barbosa E, Dozias S, Vandamme M, Cachoncinlle C, Viladrosa R and Pouvesle J 2009 Plasma Processes Polym. 6 795-802

[15] Sarron V, Robert E, Dozias S, Vandamme M, Ries D and Pouvesle J 2011 IEEE Trans. Plasma Sci. 39 2356-2357

[16] Li Q, Zhu W C, Zhu X M and Pu Y K 2010 J. Phys. D: Appl. Phys. 43382001

[17] Kirkpatrick M J, Odic E, Zinola S and Lavy J 2012 Appl. Catal. B: Environ. 117 1-9

[18] Lacoste D and Moeck J 2014 IEEE Trans. Plasma Sci. SI in press

[19] Machala Z, Chladekova L and Pelach M 2010 J. Phys. D: Appl. Phys. 43222001

[20] Babaeva N Y and Naidis G 1996 J. Phys. D: Appl. Phys. 29 2423-2431

[21] Naidis G 2009 Phys. Rev. E 79057401

[22] Pai D Z 2008 Ph.D. thesis École Centrale Paris

[23] Rusterholtz D L, Lacoste D A, Stancu G D, Pai D Z and Laux C O 2013 Journal of Physics D: Applied Physics 46464010

[24] Jansky J, Le Delliou P, Tholin F, Tardiveau P, Bourdon A and Pasquiers S 2011 J. Phys. D: Appl. Phys. 44335201

[25] Jarrige J, Laroussi M and Karakas E 2010 Plasma Sources Sci. Technol. 19065005

[26] Wu S, Wang Z, Huang Q, Tan X, Lu X and Ostrikov K 2013 Phys. Plasmas 20023503

[27] Specair 32013 www.spectralfit.com

[28] Laux C O, Spence T G, Kruger C H and Zare R N 2003 Plasma Sources Sci. Technol. 12 125-138

[29] Hofmann S, Sobota A and Bruggeman P 2012 IEEE Trans. Plasma Sci. 40 2888-2899

[30] Luque A, Ratushnaya V and Ebert U 2008 J. Phys. D: Appl. Phys. 41234005

[31] Xian Y, Lu X, Wu S, Chu P and Pan Y 2012 Appl. Phys. Lett. 100123702

[32] Benilov M and Naidis G 2003 J. Phys. D: Appl. Phys. 36 1834-1841

[33] Pancheshnyi S 2005 Plasma Sources Sci. Technol. 14 645-653

[34] Tholin F and Bourdon A 2013 Plasma Sources Sci. Technol. 22045014 


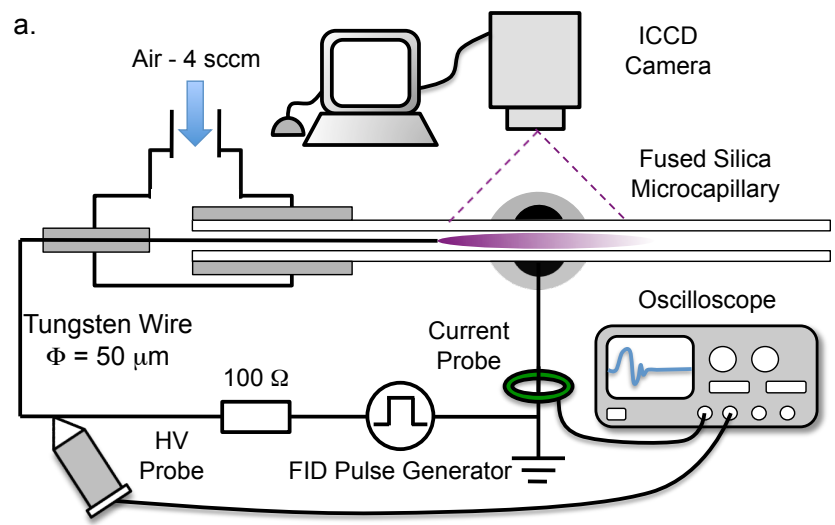

b.

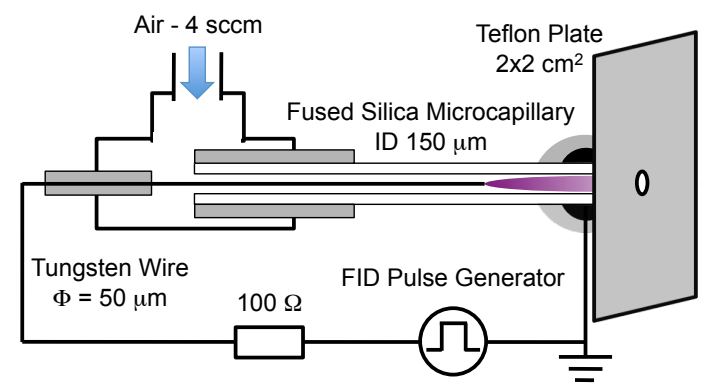

Figure 1. (a) Setup for air-plasma bullets in microcapillaries, with electrical diagnostics and ICCD camera, (b) setup for air-plasma bullets in ambient air.

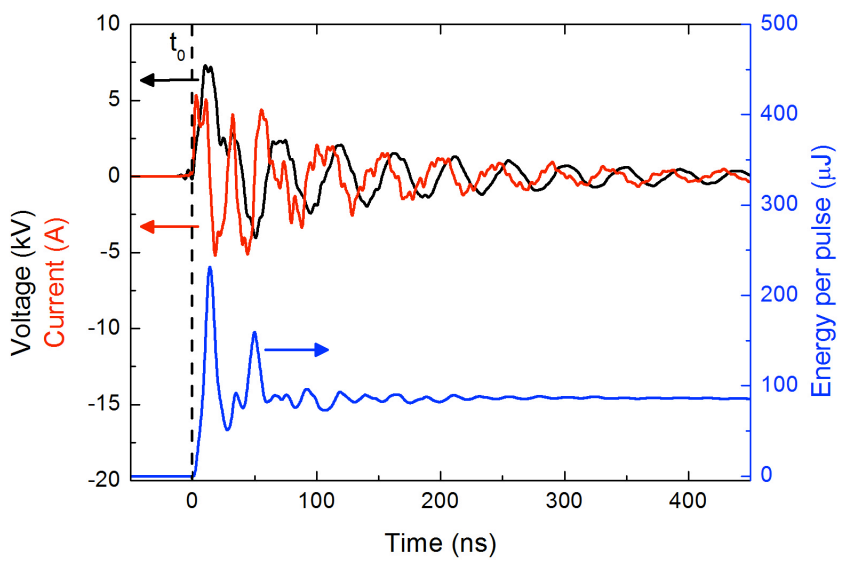

Figure 2. Example of voltage and total current measured for NRP discharges applied at $1 \mathrm{kHz}$, gap distance $1.4 \mathrm{~mm}, 150-\mu \mathrm{m}$ ID capillary, and the corresponding energy per pulse. 

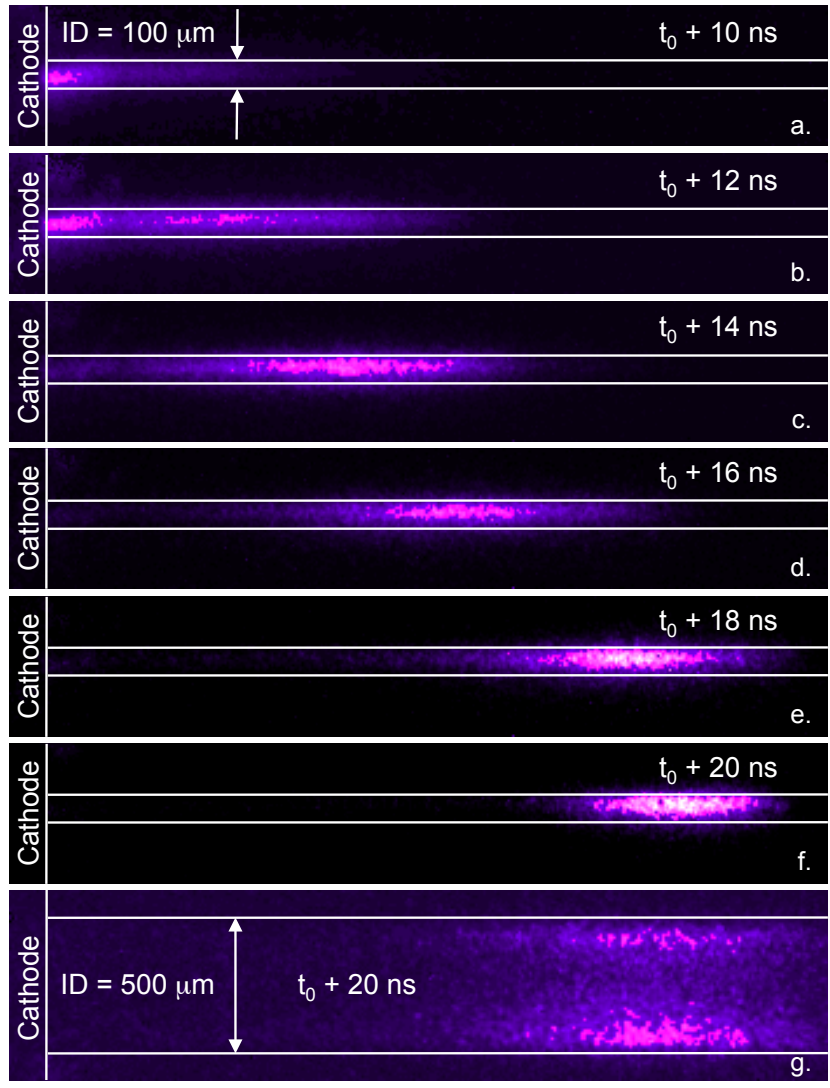

Figure 3. Visualizations of the propagation of air-plasma bullets inside a $100-\mu \mathrm{m}$ ID microcapillary (a-f), and inside a 500- $\mu \mathrm{m}$ ID microcapillary $(\mathrm{g})$, for $7.2-\mathrm{kV}$ applied voltage.

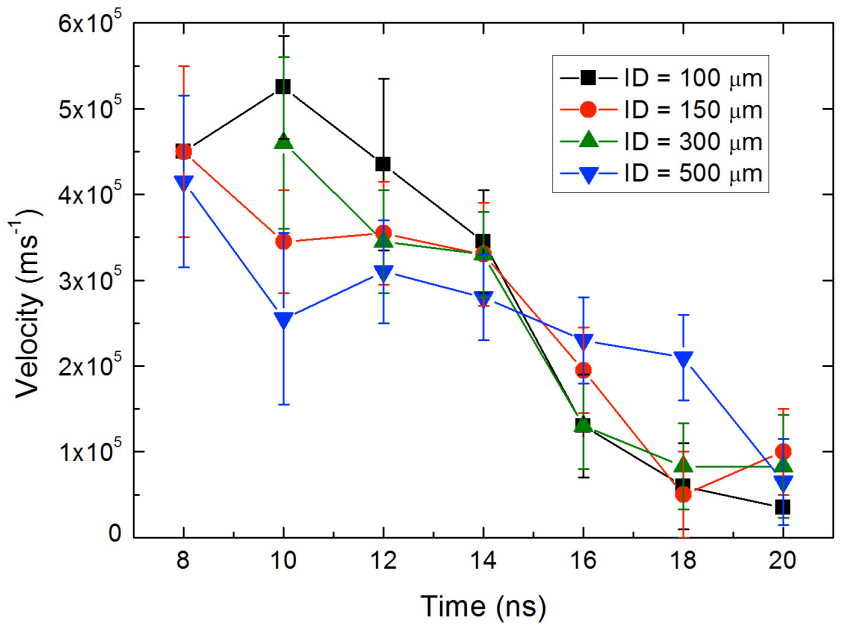

Figure 4. Influence of the inner diameter (ID) of the microcapillary on the temporal evolution of the air-plasma bullet velocity, for $8.2-\mathrm{kV}$ applied voltage. 


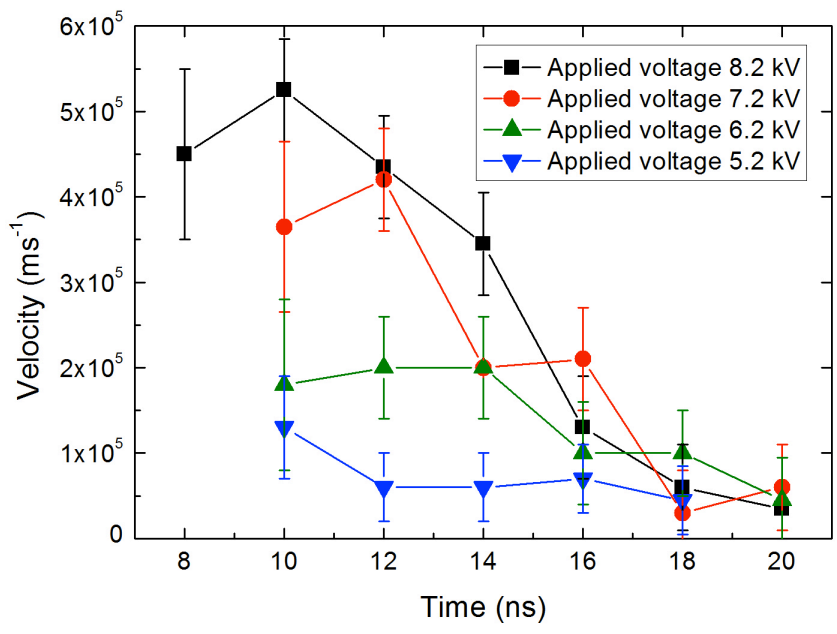

Figure 5. Influence of the applied voltage on the temporal evolution of the air-plasma bullet velocity in a $100-\mu \mathrm{m}$ ID microcapillary.

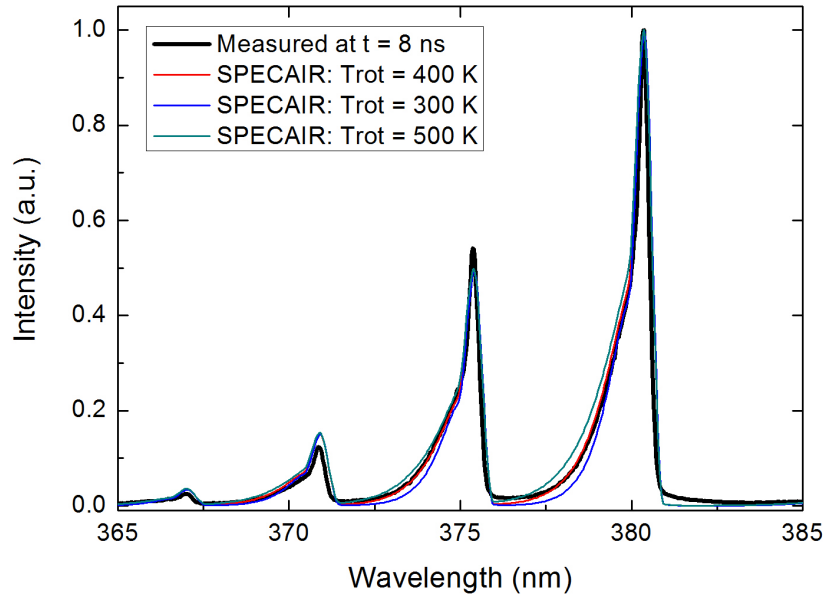

Figure 6. Example of experimental spectrum of the $\mathrm{N}_{2}(\mathrm{C}-\mathrm{B})$ transition and simulations performed with the SPECAIR software [27], for an ID of $100 \mu \mathrm{m}$ and 8.2-kV applied voltage. 


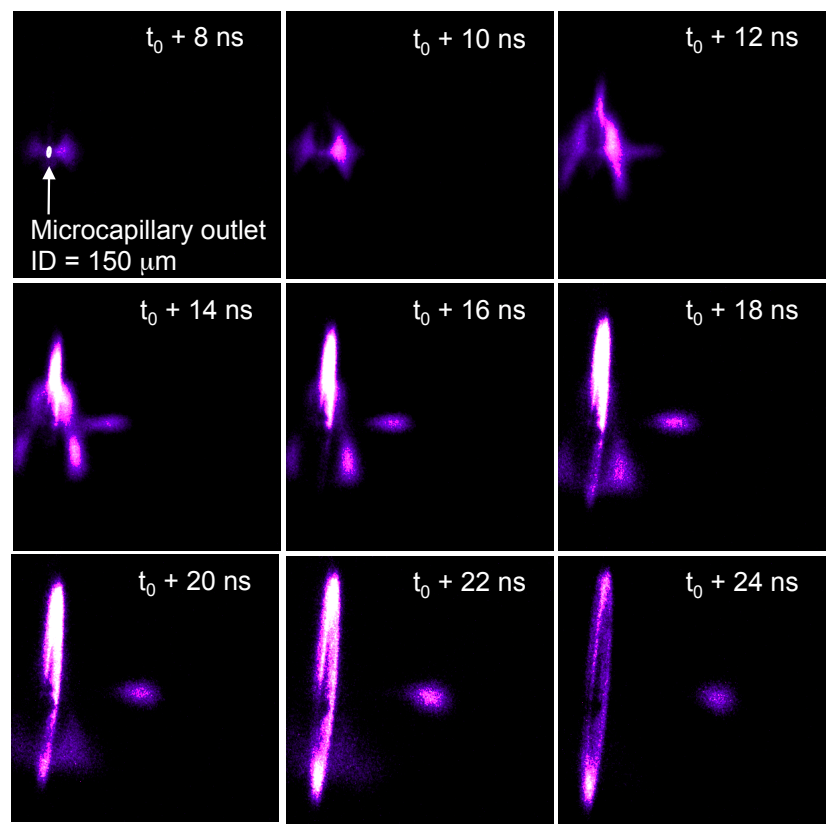

Figure 7. Visualizations of the air-plasma bullets propagating in ambient air, obtained with a $150-\mu \mathrm{m}$ ID microcapillary and $7.2-\mathrm{kV}$ applied voltage.

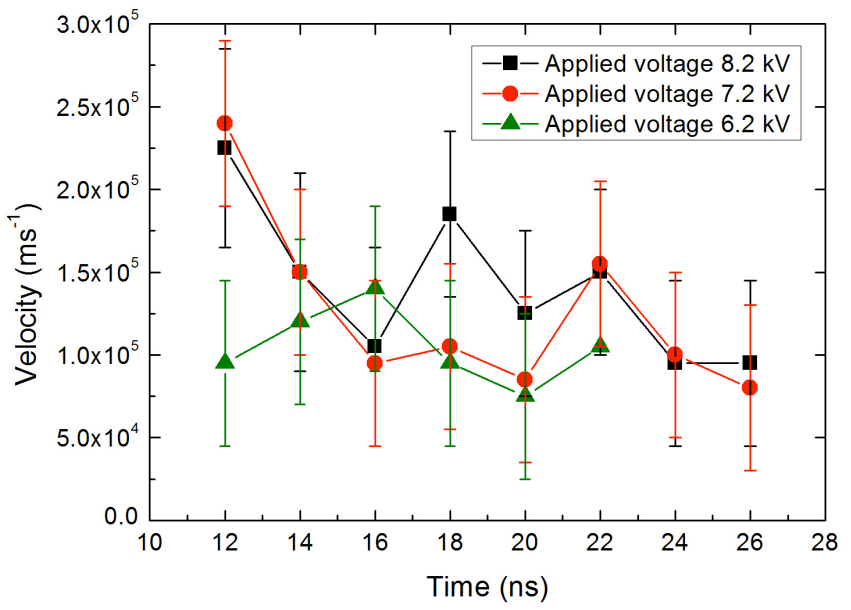

Figure 8. Influence of the applied voltage on the temporal evolution of the air-plasma bullet velocity in ambient air (150- $\mu \mathrm{m}$ ID microcapillary). 